\title{
COMPLEXES AND HOMOTOPY CHAINS
}

\section{KURT REIDEMEISTER}

The theory I have to speak about is a chapter of the algebraic topology of complexes. Its definition parallels the classical homology theory.

Let $A$ be a complex with the oriented cells $a_{\boldsymbol{t}}^{\boldsymbol{k}}$, where $k$ is the dimension number. Then the homology theory starts with the free Abelian groups of chains

$$
c^{k}=\sum \xi a^{k}, \quad \xi \in N, a^{k} \in A,
$$

generated by the $a_{\mathfrak{i}}^{\boldsymbol{k}}$ ( $N$ is the set of the integers) and the boundary homomorphism of chains

$$
\left(c^{k}\right)^{\cdot}=\sum \xi\left(a^{k}\right)^{\cdot}
$$

where

$$
\left(a_{i}^{k}\right)^{\cdot}=\sum \underset{\rho_{i j} a_{j}^{k-1}}{k}
$$

is the boundary chain of the oriented cell $a_{i}^{k}$, the $\rho_{i j}^{k}$ being the incidence numbers of the cells $a_{i}^{\boldsymbol{k}}, a_{j}^{\boldsymbol{k}-1}$. These chains and boundary matrices change by subdivision of the complex $A$, and the homology groups are the invariants with regard to this process.

The chains and boundary matrices which I introduce are defined for complexes $U$ with an adjoined group $G$ of mappings $\gamma$ of $U$ in itself, that is, of mappings

$$
\gamma u^{k}=\bar{u}^{k}
$$

of the cells $u^{k}$ of $U$ preserving the dimension, the orientation, and the incidence relations of cells. The subdivision of the euclidean plane in squares, which is mapped in itself by the group of translations with integer coefficients, is an example for a complex $U$.

The mappings $\gamma$ of the cells induce automorphisms $\gamma$ of the chains of $U$,

$$
\gamma c^{k}=\gamma \sum \xi u^{k}=\sum \xi\left(\gamma u^{k}\right),
$$

and these automorphisms commute with the boundary homomorphism; for

An address delivered before the Philadelphia Meeting of the Society, April 30, 1949, by invitation of the Committee to Select Hour Speakers for Eastern Sectional Meetings; received by the editors May 9, 1949. 


$$
\begin{aligned}
& \left(\gamma c^{k}\right)^{\cdot}=\left(\sum \xi \gamma u^{k}\right)^{\cdot}=\sum \xi\left(\gamma u^{k}\right)^{\prime}, \\
& \gamma\left(c^{k}\right)^{\cdot}=\gamma\left(\sum \xi\left(u^{k}\right)^{\cdot}\right)=\sum \xi \gamma\left(u^{k}\right)^{\cdot},
\end{aligned}
$$

and the equation $\left(\gamma u^{k}\right)^{\cdot}=\gamma\left(u^{k}\right)^{\cdot}$ holds, because $\gamma$ preserves the incidence relations.

So we can describe these chains with operators and their boundary homomorphisms by taking the elements

$$
x=\sum \xi \gamma, \quad \xi \in N, \gamma \in G,
$$

of the group ring $G N$ of $G$ as coefficients of chains. Because

$$
x c^{k}=\left(\sum \xi \gamma\right) c^{k}=\sum \xi\left(\gamma c^{k}\right)
$$

is defined, we can speak of the chains

$$
\sum x_{i} c_{i}^{k}
$$

generated by the chains $c_{i}^{k}$ and of relations

$$
\sum \bar{x}_{i} c_{i}^{k}=0
$$

between the $c_{\boldsymbol{i}}^{\boldsymbol{k}}$. And because the equation

$$
\left(x c^{k}\right)^{\cdot}=x\left(c^{k}\right)^{\cdot}
$$

holds, we can compute the boundary chains of the generated chains by the boundary chains of the generators.

If the mappings $\gamma \neq 1$ (the identity) have no fixed elements, then there exists a fundamental region $F$ of $U$ modulo $G$, namely a subset $F$ of cells $u^{k}$, so that

$$
\gamma u_{i}^{k} \quad \text { with } \gamma \in G, u_{i}^{k} \in F
$$

represent uniquely the cells of $U$. And in this case we see the groups of chains of $U$ with operators are free groups with a set $F$ of free generators $u_{i}^{k}$. Indeed the

$$
c^{k}=\sum x_{i} u_{i}^{k}=\sum \xi_{i j} \gamma_{j} u_{i}^{k}
$$

represent uniquely the chains of $U$ because $\gamma_{j} u_{i}^{\boldsymbol{k}}$ represent uniquely the cells of $U$. And the boundary homomorphism is described by a set of matrices $r_{i j}^{k}$ with coefficients in $G N$, namely the coefficients of the boundary chains of the generators expressed in these generators

$$
\left(u_{i}^{k}\right)^{\prime}=\sum r_{i j}^{k} u_{j}^{k-1}, \quad \quad r_{i j}^{k} \in G N .
$$


For the subdivision of the euclidean plane in squares we have in a fundamental region one vertex $u^{0}$, one square $u^{2}$, and two edges $u_{1}^{1}, u_{2}^{1}$, which represent two vectors of unit length, orthogonal one to the other. The group $G$ is generated by the translations $\sigma_{1}, \sigma_{2}$ determined by these vectors and we can take $F$ so that we get

$$
\begin{aligned}
& \left(u_{1}^{1}\right)^{\cdot}=-u^{0}+\sigma_{1} u^{0}, \\
& \left(u_{2}^{1}\right)^{\cdot}=-u^{0}+\sigma_{2} u^{0}, \\
& \left(u^{2}\right)^{\cdot}=u_{1}^{1}+\sigma_{1} u_{2}^{1}-\sigma_{2} u_{1}^{1}-u_{2}^{1} .
\end{aligned}
$$

Now remember a classical theorem. To every connected complex $A$ there exists a universal covering complex $U$ with a group $G$ of mappings $\gamma$ of $U$ in itself, the structure of which is uniquely determined by $A$. The group $G$ of $U$ is isomorphic to the fundamental group $G^{1}$ of $A$ defined by the homotopy classes of the paths of $A$. The mappings $\gamma$ are the deck transformations of the covering complex. That means: if $\pi$ is the projection of $U$ in $A$, which gives for every cell $u^{k}$ of $U$ the cell $a^{k}$ of $A$ which is covered by $u^{k}$, then we have: $\gamma u=\bar{u}$ implies $\pi(\bar{u})=\pi(u)$ and $\pi(\bar{u})=\pi(u)$ implies the existence of a $\gamma$ with $\bar{u}=\gamma u$. And because $\pi$ has to be faithful in the small, it follows that $\gamma u=u$ implies $\gamma=1$.

So we can define: the system of homotopy chains of the complex $A$ is the system of the chains of the universal covering complex $U$ of $A$ with the operators induced by the deck transformations of $U$. And we have the theorem: If the $u_{i}^{k}$ are a fundamental region of $U$ modulo $G$, then the $u_{i}^{\mathbf{k}}$ are free generators of the system of homotopy chains and there exists a uniquely determined set of matrices $r_{i j}^{\boldsymbol{k}}$ with elements in the group ring GN defining the boundary homomorphism of the chains in the chosen generators.

The square net of the euclidean plane is the universal covering complex of a torus $A$, the edges $a_{1}^{1}, a_{2}^{1}$ of which are singular ones beginning and ending at the same point $a^{0}$. The chains of the square net are the homotopy chains of $A$.

To finish the exposition of the theory we have to characterize algebraically the topological invariants of the matrices $r_{i j}^{k}$.

We can change the fundamental region $F$ in $\bar{F}$ by

$$
\bar{u}_{i}^{k}=\gamma_{i k} u_{i}^{k}
$$$$
\gamma \in G
$$ 
and $A$ may change by subdivision. Then $U$ is also changed by a set of induced subdivisions. This step is equivalent to a set of unimodular transformations of the generators and to the introduction of new pairs of generators $u^{k}, u^{k-1}$ with the boundary relation

$$
\left(u^{k}\right) \cdot=u^{k-1}, \quad\left(u^{k-1}\right) \cdot=0 .
$$

So we have three ways to change the set of generators. The first and the second type preserve the number of generators and by iteration of these steps we get a group of linear transformations $T$, the basis transformations, with coefficients in the group ring $G N$ which is determined in the same way for all group rings and is uniquely determined by $G N$ and the number of generators.

And the theorem holds:

If $\bar{A}$ is a complex, which is equivalent to $A$ by subdivision, then the set of the homotopy boundary matrices $\bar{r}_{i j}^{k}$ of $\bar{A}$ is equivalent to $r_{i j}^{k}$ of $A$ by basis transformations and introduction of new pairs of generators $u^{k}$, $u^{k-1}$ with

$$
\left(u^{k}\right) \cdot=u^{k-1}, \quad\left(u^{k-1}\right) \cdot=0 .
$$

Now we have to ask how to solve the equivalence problem for homotopy chain systems, and clearly the parallelism to the homology theory, which is so obvious by the definition of the equivalence, does not hold for its solution at all. There is little hope to get a method to decide if the homotopy systems of two complexes $A, \bar{A}$ are equivalent or not. For, in order to compute the boundary matrices $r_{i j}^{k}$, we need to know the fundamental group $G^{1} ; G^{1}$ is given by generators and defining relations and there exists no method to decide if two systems of generators and relations define isomorphic groups. But there exists a method to get invariants.

To be clear I define this method at first in a purely algebraic way.

Let $M$ be a ring with elements $y$ which is homomorphic to $G N$ and let

$$
\phi x=y
$$

be a homomorphism of $G N$ onto $M$. Then we get by

$$
\phi r_{i j}^{k}=\tilde{r}_{i j}^{k}
$$

a set of matrices with elements in $M$. We define the $M$-chains of $A$ by

$$
\tilde{c}^{k}=\sum y_{i} v_{i}^{k},
$$


the number of the $v_{i}^{k}$ being the same as the number of the $u_{t}^{k}$, and the boundary homomorphism by

$$
\begin{aligned}
\left(\sum y v^{k}\right) \cdot & =\sum y\left(v^{k}\right) \cdot \\
\left(v_{i}^{k}\right) \cdot & =\sum \tilde{r}_{i j}^{k} v_{j}^{k-1} .
\end{aligned}
$$

This $M$-chain system with its boundary operator is homomorphic to the homotopy chain system. Indeed the projection

$$
\phi c^{k}=\phi \sum x u^{k}=\sum \phi x \cdot v^{k}
$$

is a homomorphism of the free homotopy chain groups in the free $M$-chain groups and $\phi$ commutes with the boundary operator, because the equation

$$
\left(\phi u^{k}\right) \cdot=\phi\left(u^{k}\right)
$$

holds.

By projection of the basis transformation $T$ we get the transformation $\phi T$ for $M$-chain basis and an equivalence relation for $M$ chain systems and we see that the equivalence classes of $M$-chain systems correspond to those of the homotopy chains.

Now we may look for invariants of the $M$-chain system.

Following the classical way we define the homology group $\widetilde{H}^{k}$. Let $\tilde{Z}^{k}$ be the groups of the cycles, that is, of the chains $\tilde{z}^{k}$ with $\left(\tilde{z}^{k}\right)^{\cdot}$ $=0$ and $\widetilde{B}^{k}$ the group of the bounding chains $\tilde{b}^{k}$, that is, of the chains for which exist a chain $\tilde{b}^{k+1}$ with $\left(\tilde{b}^{k+1}\right)^{\cdot}=\tilde{b}^{k}$. Then $\left(\tilde{b}^{k}\right)^{\cdot}=0, \tilde{B}^{k} \subset \tilde{Z}^{k}$. And the $\widetilde{H}^{k}$ are defined as the factor group $\tilde{Z}^{k} / \widetilde{B}^{k}$, all these groups being groups with operators in $M$.

Observe further that the projection $\phi c^{k}$ defines a characteristic subgroup in $\tilde{H}^{k}$. Namely if $z^{k}$ is a homotopy chain with $\left(z^{k}\right)^{\cdot}=0$, then $\phi z^{k}$ is an $M$-chain with $\left(\phi z^{k}\right)^{\cdot}=0$ because $\phi$ commutes with the boundary operator, and thus we get by projection of the group $Z^{k}$ of the homotopy cycles a subgroup $\phi Z^{k}$ of $\tilde{Z}^{k}$ and, because $\phi B^{k}=\tilde{B}^{k}$, a subgroup of $\widetilde{H}^{k}$. The $\widetilde{H}^{k}$ with these characteristic subgroups $\phi Z^{k} / \widetilde{B}^{k}$ are invariants of the homotopy system.

The geometrical meaning of this algebraic construction is easy to realize for special rings $M$.

If $M=G N$, the $H^{k}$ are the homology groups of the universal covering complex with operators induced by the deck transformations. If $M=N$, and $\phi \sum \xi \gamma=\sum \xi$, the $\tilde{H}^{k}$ are the homology groups of $A$. The example of the torus shows that the homology groups of the complex $U(M=G N)$ may vanish without the homology groups of a homo- 
morphic chain system doing the same. If $M$ is a group ring of a factor group $G^{*}$ of $G$ and $\phi$ is defined by a homomorphism $\phi G=G^{*}$, then $\tilde{H}^{k}$ are the homology groups of a regular covering complex $U^{*}$ of $A$ with operators induced by the deck transformations of $U^{*}$. All these groups $\widetilde{H}^{k}$ are well known invariants of $A$, invariant also under continuous mappings of the point set complex $A$.

The characteristic subgroup $\phi Z^{k}$ is of algebraic and geometric interest for $M=N, k=2$. The existence of this group gives the argument for the remarkable theorem of Hopf that the second homology group $\widetilde{H}^{2}$ of a complex is homomorphic to a group $H^{2}\left(G^{1}\right)$, which is determined by the fundamental group $G^{1}$ of $A$. We have seen that $\widetilde{H}^{2}=\widetilde{Z}^{2} / \widetilde{B}^{2}$ is homomorphic to $\widetilde{Z}^{2} / \phi Z^{2}$ and the theorem will be proved if we show that $\tilde{Z}^{2} / \phi Z^{2}$ is determined only by $G^{1}$. Now $\tilde{Z}^{2}, Z^{2}$ are determined by the 2-dimensional subcomplex $U^{2}$ of $U$, and the $U^{2}$ is essentially determined by the generators and defining relations of the fundamental group $G^{\mathbf{1}}$.

Without loss of generality we may assume that $A$ has only one vertex $a^{0}$. Our torus complex gives an example for such a complex $A$. Then the 1 -dimensional cells $a_{\boldsymbol{k}}^{1}$ represent closed paths $\sigma_{k}$, which generate the free group $S$ of paths.

Every 2-dimensional cell $a_{j}^{2}$ has a boundary path $\rho_{j}(\sigma)$, and the set $\rho_{j}(\sigma)$ is the set of the defining relations of $G^{1}$ in the generators $\sigma_{k}$.

Otherwise if we have these generators and defining relations we can construct $A^{2}$ and with help of $A^{2}$ in the usual way the universal covering complex $U^{2}$ of $A^{2}$. The complex $U^{2}$ is the Dehn group image of $G^{1}$ with the given generators and defining relations. And we see that $\tilde{Z}^{2} / \phi Z^{2}$ is defined for every Dehn group image of $G^{1}$. It is easy to realize that $\tilde{Z}^{2} / \phi Z^{2}$ does not change if we change the generators or the defining relations and so the theorem of Hopf is proved.

The cycles $z^{2}$ may be characterized as chains induced by spheres, as Hurewicz has shown, and as chains induced by identities of defining relations, that is, products of relations $\lambda \rho \lambda^{-1}$, which equal 1 in the free group $S$, as $\mathrm{R}$. Peiffer has shown, and so we may understand the formal definition of the group $H^{2}\left(G^{1}\right)$ both geometrically and algebraically.

We may compute the boundary matrices of the 2-dimensional homotopy chain system of a group with generators and defining relations directly, without constructing $U^{2}$ and $\mathrm{R}$. Fox has shown that a process of partial derivation defined for group rings of free groups is helpful for this task. He gets the boundary matrices $r_{i j}^{2}$ as the "Jacobian" 


$$
r_{i j}^{2}=\phi \frac{\partial \rho_{i}(\sigma)}{\partial \sigma_{j}},
$$

$\phi$ being the projection of the free ring in the group ring of $G^{1}$.

Following Hopf, we can extend the construction of homotopy chain systems of a group without using a complex $U$ in higher dimension. Let $C^{3}$ be the group of the

$$
c^{3}=\sum x_{i} v_{i}^{3}, \quad x_{i} \in G N,
$$

where the

$$
\left(v_{i}^{3}\right)^{\cdot}=z_{i}^{2}, \quad z_{i}^{2} \in Z^{2},
$$

are a set of generators of $Z^{2}$. Then there exists a subgroup $Z^{3}$ of relations $z^{3}$ in the generators $v^{3}$ or of cycles with $\left(z^{3}\right)^{\circ}=0$ and the process can be iterated.

The homotopy chain systems of dimension $n$ of group $G^{1}$, which we get in that way, choosing successively sets of generators $v^{k}$, are equivalent up to the dimension $n-1$. So the groups

$$
\tilde{Z}^{k} / \phi Z^{k}
$$

are the same for all homotopy chain systems of $G^{1}$ and define homology groups $H^{k}\left(G^{1}\right)$ of higher dimension adjoined to $G^{1}$.

The homotopy chain systems of $G^{1}$ are characterized as acyclic systems up to the dimension $n-1$, that means systems in which every cycle bounds or in which the bounding cycles generate the groups of cycles. And so we get the theorem of Hurewicz, that the homology groups of a complex $A$ are determined by its fundamental group $G^{1}$ up to the dimension $n-1$, if the universal covering complex $U$ is acyclic up to the dimension $n-1$.

Hurewicz has proved this theorem with the methods of his homotopy theory. Another interesting approach in a combinatorial way is made by Eilenberg and MacLane and a little later by Eckmann, who adjoin directly to a group a combinatorial complex $U$ with a group $G$ of mappings of $U$ in itself, namely the complexes of ordered simplices the vertices of which are the group elements.

About $M$-chain systems of a group which are defined by a homomorphism $\phi G=G^{*}$, I mention that they give a natural approach to the metabelian groups and to the argument for the Haupt ideal theorem of the number theory; although this result of Schumann is not a new one, it may be of interest to see this connection between the chain systems and the groups themselves. 
Now let us go back to the $M$-chain systems of a complex $U$.

If $M$ is not a group ring we get a new type of chains, which we may define otherwise using locally groups of cells. Steenrod has extended this definition to singular chains using locally groups of points and has proved that the groups $\tilde{H}^{k}$ also for this kind of chain are invariant under topological mappings.

But the homology groups do not characterize the equivalence classes of $M$-chain systems. There exists an invariant of certain $M$ chain systems, the torsion $\tau$, which is not determined by its homology groups. We do not know if the torsion is invariant by topological mappings although we know that it is not a property of the homotopy classes of complexes. But you see the answer will be of interest in any case. And the classical question, if two complexes, which are topologically equivalent, are also equivalent by subdivision, will be answered in a negative sense, if the torsion is not a topological invariant. The torsion is defined, if $M$ is a subring of a field. We get such rings if we map the element $\boldsymbol{\gamma}$ by $\phi$ in roots of the unit

$$
\phi \gamma=e^{2 \pi i \kappa / \mu} ;
$$

$\phi \gamma$ is a so-called character of $G$ and $M$ a subring of a cyclotomic number field. The basis transformations $\phi T$ are linear transformations the determinant of which also equals a root unity, and we may ask for invariants of the boundary matrices if we admit all linear transformations of the field the determinant of which equals a root unity. Then we have to admit also new chains, namely chains with coefficients in the field and a boundary operator which commutes with the multiplication in the field. The homology groups of these chains "with division" are free groups and the torsion equals zero if one of these groups is not the identity.

If all homology groups equal the identity, then there exists a certain set of subdeterminants $d^{k}$ of the boundary matrices which are not equal to zero, and the torsion is defined by

$$
\tau=\prod \frac{d^{2 l+1}}{d^{2 l}}
$$

and we have the theorem of Franz: Two systems of boundary matrices of a field are equivalent if and only if the torsion of the two systems is the same modulo a factor of a root unity.

These algebraic calculi get geometrical interest by the fact that the torsion gives a classification of the so-called lens spaces, which are useful to show that the classical topological invariants do not 
give a full classification of complexes. The lens spaces are defined as fundamental regions of a rotation $\gamma$ of finite order $\mu$ which maps a sphere of dimension $2 \nu+1$ in itself.

Let

$$
y=\xi+i \eta, \quad \bar{y}=\xi-i \eta
$$

be complex numbers, so we get the $(2 \nu+1)$-sphere by the equation

$$
\sum_{k=0}^{\nu} y_{k} \bar{y}_{k}=1
$$

and a rotation $\gamma$ of order $\mu$ by

$$
y_{k}^{\prime}=e^{2 \pi i \lambda_{k} / \mu} y_{k}, \quad\left(\lambda_{k}, \mu\right)=1 .
$$

Then there exists a subdivision $U$ of the sphere, which is mapped by $\gamma$ in itself, and $U$ defines by projection a complex $A$ for which $U$ is the universal covering complex. Then $A$ is a complex of a lens space.

The fundamental group $G^{1}=G$ of $A$ is the cyclic group of order $\mu$ and we get a homomorphism of the group ring $G N$ in a ring $M$ of a number field by

$$
\phi \gamma=e^{2 \pi i / \mu_{0}}, \quad \quad \mu_{0} \mid \mu, \mu_{0}>2 .
$$

The torsion of this $M$-chain system of $A$ is

$$
\tau\left(A, \mu_{0}\right)=\prod\left(1-e^{2 \pi i \lambda_{k} / \mu_{0}}\right) e^{2 \pi i \lambda / \mu_{0}}
$$

and we get a purely arithmetical problem, namely to consider the multiplication groups of numbers generated by

$$
1-e^{2 \pi i \lambda / \mu_{0}}, \pm e^{2 \pi i \lambda / \mu_{0}}, \quad\left(\lambda, \mu_{0}\right)=1,
$$

and to determine the representations of the unit in these generators.

If $\mu$ is a prime number, this question is answered by the theorem that the circle units of Hilbert generate a free group. If $\mu$ is not a prime number we have to argue (following Franz) a generalization of this theorem using the $L$-series $L(s, \phi \gamma)$ adjoined to the characters $\phi \gamma$. In both cases we realize that if the equation

$$
\tau\left(A, \mu_{0}\right)=\tau\left(A^{\prime}, \mu_{0}\right)
$$

holds, the two sets of numbers $\left( \pm \lambda_{k}\right),\left( \pm \lambda_{k}^{\prime}\right)$ are the same.

But then we go from $A^{\prime}$ to $A$, changing the enumerating of the $\lambda$ (or the $y$ ) and changing some $\lambda$ in $-\lambda$ (or some $y$ in $\bar{y}$ ), and so it fol- 
lows that lens spaces with the same torsion are equivalent under subdivision.

The fact that $\tau$ is not a property of the homotopy classes follows from the homology classification of the lens spaces, which L. W. Franz, J. H. C. Whitehead and Olum have given, the latter using a homotopy invariant defined by Eilenberg and MacLane.

You may foresee that it is possible to combine the notions of intersection, of cohomology and products of chains and cochains with the operator process $\gamma$. The development of the operator calculus in this direction given by Burger, Eckmann, Eilenberg, MacLane, Steenrod, I can only mention. Computing the duality properties of homotopy chain systems, we get for 3-dimensional manifolds a condition for the fundamental group $G^{1}$; if $G^{1}$ is abelian, $G^{1}$ is either cyclic or the free abelian group with 3 generators. Last but not least we can adjoin to a mapping $f: A \rightarrow A$ a class of mappings $f_{u}: U \rightarrow U$ and then a set of linear transformations of the homotopy chains. Thus we get a generalized Lefschetz trace formula and Wecken has proved that this trace invariant gives the exact minimal number of fixed points of a deformation class of the mappings $f: A \rightarrow A$, if the dimension of $A$ is greater than 2 .

\section{BIBLIOGRAPHY}

1. B. Eckmann, Der Cohomologiering einer beliebigen Gruppe, Comment. Math. Helv. vol. 18 (1945-1946) p. 232.

2. S. Eilenberg and S. MacLane, Relations between homology and homotopy groups of spaces, Ann. of Math. vol. 46 (1945) p. 489.

3. - Cohomology theory in abstract groups I, Ann. of Math. vol. 48 (1947) p. 51.

4. S. Eilenberg, Homology of spaces with operators, Trans. Amer. Math. Soc. vol. 61 (1947) p. 378.

5. W. Franz, Über die Torsion einer Überdeckung, J. Reine Angew. Math. vol. 173 (1935) p. 245.

6. - Überdeckungen topologische Komplexe mit hyperkomplexen Systemen, ibid. p. 174.

7. H. Hopf, Fundamentalgruppe und zweite Bettische Gruppe, Comment. Math. Helv. vol. 14 (1941-1943) p. 257.

8. - U Über die Bettischen Gruppen, die zu einer beliebigen Gruppe gehören, Comment. Math. Helv. vol. 17 (1944-1945) p. 39.

9. W. Hurewicz, Beitrage zur Topologie der Deformationen, Akademie van Wetenschappen Amsterdam, Proceedings of the Section of Sciences vol. 38 (1935) pp. 112, 521 and vol. 39 (1936) p. 215.

10. K. Reidemeister, Topologie der Polyeder, Leipzig, 1938, p. 177.

11. - Überdeckungen von Komplexen, J. Reine Angew. Math. vol. 173 (1935) p. 164 .

12. - Kommutative fundamental Gruppen, Monatshefte für Mathematik und Physik vol. 43 (1936) p. 20. 
13. H. G. Schumann, Über Moduln und Gruppenbilder, Math. Ann. vol. 114 (1937) p. 385.

14. N. E. Steenrod, Homology with local coefficients, Ann. of Math. vol. 44 (1943) p. 610.

15. F. Wecken, Fixpunkt klassen, Math. Ann. vol. 117 (1939-1941) p. 659, vol. 118 (1941-1943) pp. 216, 544.

16. J. H. C. Whitehead, On the homotopy type of manifolds, Ann. of Math. vol. 41 (1940) p. 825.

UNIVERSITY OF MARBURG AND

INSTITUTE FOR ADVANCEd STUdy 